\title{
ATTEMPTS TO DETECT NEUTRAL HYDROGEN \\ IN COMPACT OBJECTS
}

\author{
WILLIAM A. DENT \\ University of Massachusetts, Amherst, Mass., U.S.A.*
}

\begin{abstract}
An attempt was made to detect the redshifted $\mathrm{HI}$ line in absorption in the quasi-stellar sources $3 C 273$ and $1510-08$ and in the N-galaxies 3C 120 and 3C 371. The Seyfert galaxies NGC 1068 and NGC 1275 were also searched for $\mathrm{HI}$ in absorption as well as in emission. Although no lines were detected, it was possible to place upper limits on the column density of $\mathrm{HI}_{\mathrm{I}}$ in all these objects, as well as upper limits on the mass of $\mathrm{HI}_{\mathrm{I}}$ in the Seyfert galaxies observed.
\end{abstract}

\section{Introduction}

The existence of intense, compact and frequently variable radio components in quasistellar sources, Seyfert galaxies, and N-type galaxies suggests the possibility of detecting neutral hydrogen in these objects by observing it in absorption against the intense continuum radio source present.

Following the suggestion of Bahcall and Ekers (1969), Shuter and Gower (1969) and Heiles and Miley (1970) have, without success, attempted to detect $\mathrm{HI}_{\mathrm{I}}$ in absorption in a few distant $(z \sim 2)$ quasars which have optical absorption lines. This paper presents the results of a search for HI absorption in nearby variable radio sources where the probability of such a detection may be higher than for more distant objects.

Detection of an $\mathrm{HI}$ absorption line is more likely in those objects which are known to contain at least one variable radio component whose emission extends to frequencies near $1420 \mathrm{MHz}$. Such components, besides providing an intense background continuum source, have linear dimensions on the order of a few parsec or less and hence could be occulted by a Hi cloud of comparable size or larger within the object.

From the observed differences between optical absorption and emission line redshifts the expected frequency of an $\mathrm{HI}_{\mathrm{I}}$ absorption line would not necessarily be found at the frequency $1420.4 /\left(1+z_{\text {em }}\right) \mathrm{MHz}$ when only an optical emission line redshift is observed. However it is unlikely that as large a difference between emission and absorption redshifts would exist in Seyfert and $\mathrm{N}$-type galaxies as in highly redshifted quasars. ${ }^{* *}$ The absorption redshift in $1510-08$, one of the two quasars, observed in this paper, is 0.358 compared with an emission line redshift of 0.361 (Burbidge and Kinman, 1966). This difference corresponds to only about $1 \mathrm{MHz}$ at the redshifted frequency of the $21-\mathrm{cm} \mathrm{HI}$ line. Thus it is felt that no absorption lines are likely to be found outside the actual frequency interval (up to $14 \mathrm{MHz}$ ) that was searched in each object.

* Contribution from the Four College Observatories, No. 89.

** Roberts (1970) has recently reported the existence of an $\mathrm{HI}$ absorption line in the nearby radio galaxy NGC 5128 that differs in radial velocity from that of the optical emission lines by only $23 \mathrm{~km} \mathrm{~s}^{-1}$. 
All except one of the objects observed (1510-08) have no observed optical absorption lines. The presence of absorption is not necessarily an important criterion since most of the strong resonances absorption lines seen in quasars are found at frequencies that are redshifted into the visible region in only high redshifted objects.

The observations were made with the $140-\mathrm{ft}$ ( $43 \mathrm{~m}$ ) antenna of the National Radio Astronomy Observatory using the 413 channel auto-correlation spectral line receiver at its maximum bandwidth of $10 \mathrm{MHz}$ and with a resolution of $52 \mathrm{kHz}$. Three different parametric amplifier front-ends were used to observe sources in the range 1040 to $1420 \mathrm{MHz}$.

A load switched on-source, off-source observational procedure was used to remove first order instrumentally-produced spectral slopes. Second order spectral effects such as that due to the source continuum emission, uncertainties in the gain calibration resulting from extensive man-made interference outside the protected frequency bands, and radiated emission reflected from the surface of the disk were minimized as much as possible. The details of the observations and the above calibration procedures will be published elsewhere (Dent, 1971).

\section{HI in Absorption}

No obvious absorption lines were found in any of the objects studied. There may be a suggestion of a line near $1397 \mathrm{MHz}$ in NGC 1275; however, the signal to noise ratio is not adequate to claim a positive detection. Observations with greater sensitivity are planned for this source.

Upper limits to the depth of the line, $\Delta T_{L}$, and the ratio of line depth to continuum antenna temperature, $\Delta T_{L} / T_{C}$, are given for each source in Table I and represent the principle result of these observations. The quoted upper limits of $\Delta T_{L}$ are one-half the peak-to-peak noise fluctuations.

If the $\mathrm{HI}_{\mathrm{I}}$ in the object is seen against the background of a continuum source, with

TABLE I

\begin{tabular}{|c|c|c|c|c|c|c|}
\hline Source & $\begin{array}{l}\text { Type of } \\
\text { object }\end{array}$ & $z$ (ref) & $\begin{array}{l}\text { Frequencies } \\
\text { searched (MHz) }\end{array}$ & $\Delta T_{L}(\mathrm{~K})$ & $\Delta T_{L} / T_{C}$ & $\mathrm{~N}_{\mathrm{HI}}\left(\mathrm{cm}^{-2}\right)^{\mathrm{a}}$ \\
\hline $\begin{array}{l}\text { NGC } 1068 \\
(3 C 71)\end{array}$ & Seyfert & 0.0037 (1) & $1410-1420$ & $<0.075$ & 0.052 & $<2 \times 10^{21}$ \\
\hline $\begin{array}{l}\text { NGC } 1275 \\
(3 C 84)\end{array}$ & Seyfert & $0.0176(2)$ & $1392-1403$ & $\leqslant 0.157$ & 0.042 & $\leqslant 2 \times 10^{21}$ \\
\hline $3 C 120$ & N-Gal & $0.0334(3)$ & $1370-1382$ & $<0.051$ & 0.039 & $<2 \times 10^{21}$ \\
\hline $3 C 371$ & N-Gal & 0.050 & $1348-1360$ & $<0.070$ & 0.093 & $<4 \times 10^{21}$ \\
\hline $3 C 273$ & QSS & 0.158 & $1220-1234$ & $<0.089$ & 0.007 & $<3 \times 10^{22}$ \\
\hline $1510-08$ & QSS & 0.361 (6) & $1040-1053$ & $<0.068$ & 0.080 & $<3 \times 10^{23}$ \\
\hline
\end{tabular}

a Assuming $\Delta v \leqslant 100 \mathrm{KHz} ; T_{S} \approx 10^{3} \mathrm{~K}$ for Seyfert and N-Galaxies and $T_{S} \approx 10^{5} \mathrm{~K}$ for quasars. References: (1) Walker (1968); (2) Burbidge and Burbidge (1965); (3) Burbidge (1967); (4) Sandage (1966); (5) Schmidt (1963); (6) Burbidge and Kinman (1966). 
brightness temperature $T_{B}$, and if $T_{B}>T_{S}$, the spin (excitation) temperature of the $\mathrm{HI}$, then the line will appear in absorption with the depth of a line, $\Delta T_{L}$ given by

$$
\Delta v \Delta T_{L} / T_{C}=2.85 \times 10^{-15} N_{\mathrm{HI}} / T_{S}
$$

where $\Delta v$ is the effective line width in $\mathrm{Hz}$, and $N_{\mathrm{HI}}$ is the column density of $\mathrm{HI}$ in $\mathrm{cm}^{-2}$. Thus the ratio $N_{\mathrm{HI}} / T_{S}$ can be determined from the observational quantities on the left side of the equation.

Because the spin temperature of the neutral hydrogen in these sources is not accurately known, only crude estimates of the upper limit to the Hi column densities can be made using a calculated value of $T_{S}$. However, since the contributions to $T_{S}$ from Lyman $\alpha$ and $21-\mathrm{cm}$ photons depend as $r^{-2}$ upon the distance of the Hi from the photon sources, it is also necessary to assume an average distance of any $\mathrm{HI}$ present from these emitting regions within the object. If we adopt a plausible distance of $10^{4} \mathrm{pc}$; and use measurements of the radio continuum flux, estimates of the Ly $\alpha$ flux (Wampler, 1968) and expressions for $T_{S}$ given by Bahcall and Ekers (1969); we obtain rough estimates of $T_{S} \approx 10^{3} \mathrm{~K}$ in Seyfert and $\mathrm{N}$-type galaxies and $T_{S} \approx 10^{5} \mathrm{~K}$ in quasars.

Bahcall and Ekers (1969) have argued that the absence of absorption lines of OI $\lambda 1303.5$ or $N_{I} \lambda 1134.6,1199.9$ in the optical spectra of large redshifted quasi-stellar sources implies that the $\mathrm{HI}_{\mathrm{I}}$ line (if it exists) must have line widths less than about $100 \mathrm{kHz}$. Although the $\mathrm{OI}_{\mathrm{I}}$ and NI lines would not be redshifted into the visible in the objects studied in this paper, similar excitation and ionization conditions probably exist in these objects as well. Thus in the absence of a positive detection of a line, we will adopt a value of $\Delta v \leqslant 100 \mathrm{kHz}$ for the purposes of calculation.

Assuming this upper limit of $\Delta v$ and the above estimates of $T_{S}$, upper limits to the column density of neutral hydrogen were calculated and are tabulated in the last column of Table I. It should be emphasized that because of the large uncertainties in estimating $T_{S}$, the quoted upper limits to $N_{\mathrm{HI}}$ may be uncertain by more than an order of magnitude.*

\section{HI in Emission}

The 21-cm Hi line will appear in emission from those regions of the object in which the spin temperature is greater than the background brightness temperature of the continuum emission. The $\mathrm{HI}$ line should therefore appear in emission everywhere in the object except directly in front of the small intense radio component. Since none of the objects observed here could be resolved with the $20^{\prime}$ beamwidth of the antenna, only an effective antenna temperature, $\Delta T_{L}(\mathrm{em})$, of the emission line integrated over the object could be observed. It is easily shown that an estimate of the total mass of $\mathrm{HI}_{\mathrm{I}}$ in the object can be obtained from $\left.M_{\mathrm{HI}}=3.1 \times 10^{-6} R^{2}\left(\lambda^{2}\right) / A_{e}\right) \Delta T_{L}(\mathrm{em}) \Delta v$ (solar

* It should be mentioned that the value of $T_{C}$ used in the calculation is the total source antenna temperature consisting of contributions from the small intense components as well as emission from more extended regions. Thus depending on the location of the $\mathrm{HI}$, it may be necessary to adjust further the limits of $N_{\mathrm{HI}}$. 
masses), where $R$ is the distance to the source in parsecs, $A_{e}$ is the effective area of the antenna, $\lambda$ the wavelength, and $\Delta v$ the effective line width in $\mathrm{Hz}$.

In normal spiral galaxies $\Delta v$ is a Doppler width due to the rotation of the galaxy, and is on the order of $200 \mathrm{~km} \mathrm{~s}^{-1}$ or about $1 \mathrm{MHz}$ at $1420 \mathrm{MHz}$. Neutral hydrogen emission in these objects would be expected to have similar line widths since hydrogen with much higher velocities is likely to be almost completely ionized in these objects. The spectra were smoothed with a $500 \mathrm{kHz}$ gaussian filter and examined for possible emission lines. Although no obvious lines were found, meaningful upper limits on $\Delta T_{L}(\mathrm{em})$ were obtained for the two Seyfert galaxies. Upper limits on $\Delta T_{L}(\mathrm{em})$ of $0.05 \mathrm{~K}$ and $0.06 \mathrm{~K}$ were obtained for NGC 1068 and NGC 1275 respectively. Assuming a Hubble constant of $100 \mathrm{~km} \mathrm{~s}^{-1} \mathrm{Mpc}^{-1}$ and $\Delta v=1 \mathrm{MHz}$, these values yield upper limits to the masses of $1 \times 10^{9} M_{\odot}$ and $3 \times 10^{10} M_{\odot}$ respectively. Because the upper limits to the masses are proportional to $R^{2}$ the remaining four more distant objects do not give meaningful upper limits to the mass of $\mathrm{H}$.

The most recent estimate (Walker, 1968) of the mass of the inner region $(<2 \mathrm{kpc})$ of NGC 1068 is $6 \times 10^{9} M_{\odot}$. An earlier study by Burbidge et al. (1959) which did not assume a non-rotational component of the gas obtained a higher figure of $2 \times 10^{10} M_{\odot}$ for the inner region. An estimate of the total mass of the entire galaxy would be at least a factor of 2 to 4 times greater than the mass obtained for the inner region (Roberts, 1969). Hence a lower limit to the total mass of NGC 1068 is about $4 \times 10^{10} M_{\odot}$. Thus the upper limit of $1 \times 10^{9} M_{\odot}$ to the mass of neutral hydrogen in NGC 1068 (3C 71) obtained from these observations places an upper limit on the ratio $M_{\mathrm{HI}} / M_{T}$ of 0.025 . This value is less than the average value of 0.05 (Roberts, 1969) for $M_{\mathrm{HI}_{\mathrm{I}}} / M_{T}$ of Sb galaxies, the classification given to NGC 1068 by Humason et al. (1956) on the basis of its spiral structure.

Thus although the Seyfert galaxy NGC 1068 shows some similarities to normal spirals, it may be under-abundant in neutral hydrogen. This would not be surprising in view of the large flux of ionizing radiation likely to be emanating from the nucleus of this galaxy.

\section{Acknowledgements}

I would like to thank the staff of the National Radio Astronomy Observatory for making these observations possible. This work was supported in part by a Faculty Research Grant from the University of Massachusetts and by the National Science Foundation under grant number GP-14690.

\section{References}

Bahcall, J. N. and Ekers, R. D.: 1969, Astrophys. J. 157, 1055.

Burbidge, E. M.: 1967, Astrophys. J. Letters 149, L51.

Burbidge, E. M. and Burbidge, G. R.: 1965, Astrophys. J. 142, 1351.

Burbidge, E. M., Burbidge, G. R., and Prendergast, K. H.: 1959, Astrophys. J. $130,26$.

Burbidge, E. M. and Kinman, T. D.: 1966, Astrophys. J. 145, 654.

Dent, W. A.: 1971, Astrophys. J. 165, 451. 
Heiles, C. and Miley, G. K.: 1970, Astrophys. J. Letters 160, L83.

Humason, M. L., Mayall, N. U., and Sandage, A. R.: 1956, Astron. J. 61, 97.

Roberts, M. S.: 1969, Astron. J. 74, 859.

Roberts, M. S.: 1970, Astrophys. J. Letters 161, L9.

Sandage, A.: 1966, Astrophys. J. 145, 1.

Schmidt, M.: 1963, Nature 197, 1040.

Shuter, W. L. H. and Gower, J. F. R.: 1969, Nature 223, 1046.

Walker, M. F.: 1968, Astrophys. J. 151, 71.

Wampler, J.: 1968, Astrophys. J. 144, 443. 